\title{
Targeting the posterior subthalamic area for essential tremor: proposal for MRI-based anatomical landmarks
}

\author{
Andreas Nowacki, MD, Ines Debove, MD, ${ }^{2}$ Frédéric Rossi, MD,, Janine Ai Schlaeppi, MD, \\ Katrin Petermann, MSc, ${ }^{2}$ Roland Wiest, MD, ${ }^{3}$ Michael Schüpbach, MD, ${ }^{2}$ and Claudio Pollo, MD ${ }^{1}$ \\ Departments of ${ }^{1}$ Neurosurgery, ${ }^{2}$ Neurology, and ${ }^{3}$ Diagnostic and Interventional Neuroradiology, Inselspital, University Hospital \\ Bern, and University of Bern, Bern, Switzerland
}

\begin{abstract}
OBJECTIVE Deep brain stimulation (DBS) of the posterior subthalamic area (PSA) is an alternative to thalamic DBS for the treatment of essential tremor (ET). The dentato-rubro-thalamic tract (DRTT) has recently been proposed as the anatomical substrate underlying effective stimulation. For clinical purposes, depiction of the DRTT mainly depends on diffusion tensor imaging (DTI)-based tractography, which has some drawbacks. The objective of this study was to present an accurate targeting strategy for DBS of the PSA based on anatomical landmarks visible on MRI and to evaluate clinical effectiveness.
\end{abstract}

METHODS The authors performed a retrospective cohort study of a prospective series of $11 \mathrm{ET}$ patients undergoing bilateral DBS of the PSA. The subthalamic nucleus and red nucleus served as anatomical landmarks to define the target point within the adjacent PSA on 3-T T2-weighted MRI. Stimulating contact (SC) positions with reference to the midcommissural point were analyzed and projected onto the stereotactic atlas of Morel. Postoperative outcome assessment after 6 and 12 months was based on change in Tremor Rating Scale (TRS) scores.

RESULTS Actual target position corresponded to the intended target based on anatomical landmarks depicted on MRI. The total TRS score was reduced (improved) from $47.2 \pm 15.7$ to $21.3 \pm 10.7(p<0.001)$. No severe complication occurred. The mean SC position projected onto the PSA at the margin of the cerebellothalamic fascicle and the zona incerta.

CONCLUSIONS Targeting of the PSA based on anatomical landmarks representable on MRI is reliable and leads to accurate lead placement as well as good long-term clinical outcome.

https://thejns.org/doi/abs/10.3171/2018.4.JNS18373

KEYWORDS DBS; essential tremor; posterior subthalamic area; functional neurosurgery; targeting

$\mathrm{D}$ EEP brain stimulation (DBS) is an effective treatment for essential tremor (ET) in patients who do not tolerate or respond to medication. ${ }^{20}$ The classical target for DBS treatment of ET is the ventrolateral thalamus (or ventral intermediate nucleus [Vim] according to Hassler $\left.{ }^{15}\right){ }^{4}$ Thalamic DBS has been demonstrated to improve tremor severity up to $48 \%-57 \%$. $^{3,14,19,29}$ However, the optimal site is still a matter of debate, and other targets have been suggested for both lesioning and DBS. The posterior subthalamic area (PSA) includes the zona incerta (ZI) and the prelemniscal radiation (RaprL), which itself consists of the cerebellothalamic fascicle (fct) (or dentato-rubro-thalamic tract [DRTT]) and pallidothalamic fibers, and was targeted for lesioning as early as 1960 by Wertheimer et al..$^{39}$ Since then, several studies of DBS of this region have been published, demonstrating very good results, with up to $80 \%$ symptom reduction., ${ }^{2,17,26,32,33}$ However, analysis of the active or stimulating contact (SC) position revealed that stimulation sites within the PSA differed considerably between different studies. ${ }^{12}$ More recently, the DRTT has been suggested as the anatomical substrate for stimulation-induced alleviation of tremor on the basis of deterministic and probabilistic diffusion tensor imaging (DTI) studies. ${ }^{8,9,37}$ Anatomical data confirm the close anatomical relationship between the DRTT and the PSA and ZI. ${ }^{8,9}$ However, DTI has some technical limitations with an impact on accuracy of the depicted fiber tracts: due to its low signal-to-noise ratio, relatively large voxel sizes $(2-3 \mathrm{~mm})$ have to be selected in clinical practice, which has implications for the depiction of small fiber

ABBREVIATIONS AP = anteroposterior; DBS = deep brain stimulation; DRTT = dentato-rubro-thalamic tract; DTI = diffusion tensor imaging; ET = essential tremor; fct = cerebellothalamic fascicle; $\mathrm{LAT}=$ lateral; $\mathrm{MER}=$ microelectrode recording; $\mathrm{MCP}=$ midcommissural point; PSA = posterior subthalamic area; RaprL = prelemniscal radiation; $\mathrm{ROI}$ = region of interest; SC = stimulating contact; STN = subthalamic nucleus; TRS = Tremor Rating Scale; VERT = vertical; Vim = ventral intermediate nucleus; ZI = zona incerta.

SUBMITTED February 12, 2018. ACCEPTED April 12, 2018.

INCLUDE WHEN CITING Published online October 12, 2018; DOI: 10.3171/2018.4.JNS18373. 
tracts such as the DRTT. ${ }^{1}$ Additionally, the tensor calculation fails to display crossing fibers within the same voxel, which is a known feature of the DRTT. ${ }^{25}$ Thus, a targeting strategy based on DTI-based tractography might lead to inaccurate results.

At this stage, no systematic approach based on MRIdepictable landmarks to target the PSA has been proposed. Motivated by the excellent results of PSA DBS reported by different groups and the inhomogeneity of targeting approaches across these studies, our objective in the current work is to present a systematic and reproducible targeting strategy for DBS of the PSA based on anatomical landmarks. We were interested in the reliability of the targeting method and its long-term clinical effectiveness in patients treated with bilateral DBS. Furthermore, we analyzed the anatomical position of the stimulation site as well as its relation to DTI tractography results to evaluate the proposed current concept of the DRTT as the underlying neural structure responsible for the tremor-suppressive effect of DBS in patients with ET.

\section{Methods \\ Patients}

This is a retrospective, single-center study of a series of 11 consecutive ET patients (7 male, 4 female; mean age 67 \pm 14 years) who underwent bilateral DBS of the PSA, with the DBS units being implanted between June 2014 and December 2016. Eligibility criteria based on ET diagnosis were assessed by specialized movement disorder neurologists according to the accepted guidelines..$^{10}$ Patients were discussed by a multidisciplinary team and deemed suitable candidates for surgery if 1) they had severe, functionally disabling tremor impairing activities of daily living and quality of life, 2) they did not tolerate or respond to medical treatment (i.e., propranolol or primidone), and 3 ) they had no contraindications for surgery. No patients were excluded from the final analysis. The study was approved by the local ethics review board.

\section{Target Planning}

Preoperative imaging was performed with a 3-T MRI system (MAGNETOM Trio Tim, Siemens). A standard gadolinium-enhanced T1-weighted protocol (160 sagittal slices, 1-mm thickness) was followed by T2-weighted sequences (FOV $220 \mathrm{~mm}$, acquisition matrix $128 \times 128$, TR $2000 \mathrm{msec}$, and multiple TE values ranging from $12 \mathrm{msec}$ to $96 \mathrm{msec}$ in steps of $12 \mathrm{msec}$ ) and DTI (number of gradient directions 12, 2.2-mm slice thickness; 55 slices, TR $10,100 \mathrm{msec}$, TE $88 \mathrm{msec}$, field of view $280 \mathrm{~mm}$, matrix $256 \times 256$, b value $1305 \mathrm{sec} / \mathrm{mm}^{2}$ ). Target planning was based on iPlan NET software 3.0 (Brainlab AG).

The target was defined visually on T2-weighted axial slices based on anatomical landmarks (Fig. 1A and B): a horizontal line was constructed through the equator of the red nucleus at the level of its maximum diameter. A second line was drawn from the most anteromedial to the most posterolateral point of the subthalamic nucleus (STN), representing the STN axis. A third line, perpendicular to the axis of the STN, was constructed to cross the first line at the lateral border of the red nucleus. The target point was selected on that third perpendicular line halfway between the lateral border of the red nucleus and the medial border of the STN. The entry point was selected near the coronal suture. Trajectories were planned to avoid crossing sulci, blood vessels, and the ventricles.

A Leksell G frame (Elekta Instruments) was placed, and a high-resolution, stereotactic CT scan was performed and co-registered with the preoperative MRI.

\section{Electrophysiology Protocol and Intraoperative Clinical Testing}

Patients were operated on under local anesthesia. Intraoperative microelectrode recording (MER) (FHC microTargeting electrodes) and clinical testing were performed as described recently. ${ }^{27}$ Recording was typically performed on 3 parallel trajectories (central, anterolateral, and posteromedial). From 10 to $5 \mathrm{~mm}$ above the target, electrophysiological activity was recorded in 1-mm steps, and from $5 \mathrm{~mm}$ above the target it was recorded in 0.5 $\mathrm{mm}$ steps. Specialized movement disorder neurologists analyzed the electrophysiological pattern. The trajectory without electrophysiological STN activity (typically marked by increased baseline signal amplitude and sustained irregular neuronal activity with increased spike frequency, as well as bursting cells) that yielded the best intraoperative clinical testing results was used for implantation of the permanent DBS lead. The DBS lead model Activa 3389 (Medtronic) was implanted in 7 patients, and the directional DBS lead model Boston Vercise (Boston Scientific) was implanted in 5 patients.

\section{Fiber Tracking}

DTI sequences were automatically corrected for eddy current distortions and head motion. Deterministic fiber tractography was performed using Brainlab software iPlan NET 3.0 by applying 3 different regions of interest (ROIs): the manually segmented dentate nucleus of the ipsilateral cerebellum, a second manually segmented ipsilateral region in the PSA defined on T2-weighted imaging, and a cubic box including the ipsilateral precentral gyrus according to the Yousry criteria ${ }^{40}$ The minimum fiber length was set at $30 \mathrm{~mm}$, and the fractional anisotropy (FA) value was adjusted to display 1 homogeneous fiber bundle included in both ROIs.

\section{Measurements}

A postoperative high-resolution $\mathrm{CT}$ scan was performed and fused to the preoperative target plan. The postoperative lead tip and SC position as well as the vector of error were determined as described previously by our group..$^{28}$ Images were reconstructed according to the anterior commissure-posterior commissure plane. The distance between the center of the SC and the center of the DRTT tractography model was measured in each individual patient (Fig. 1C and D).

\section{Atlas- and MRI-Based Anatomy Analysis}

The lateral (LAT), anteroposterior (AP), and vertical (VERT) stereotactic coordinates of each DBS lead tip as well as each SC with reference to the midcommissural 

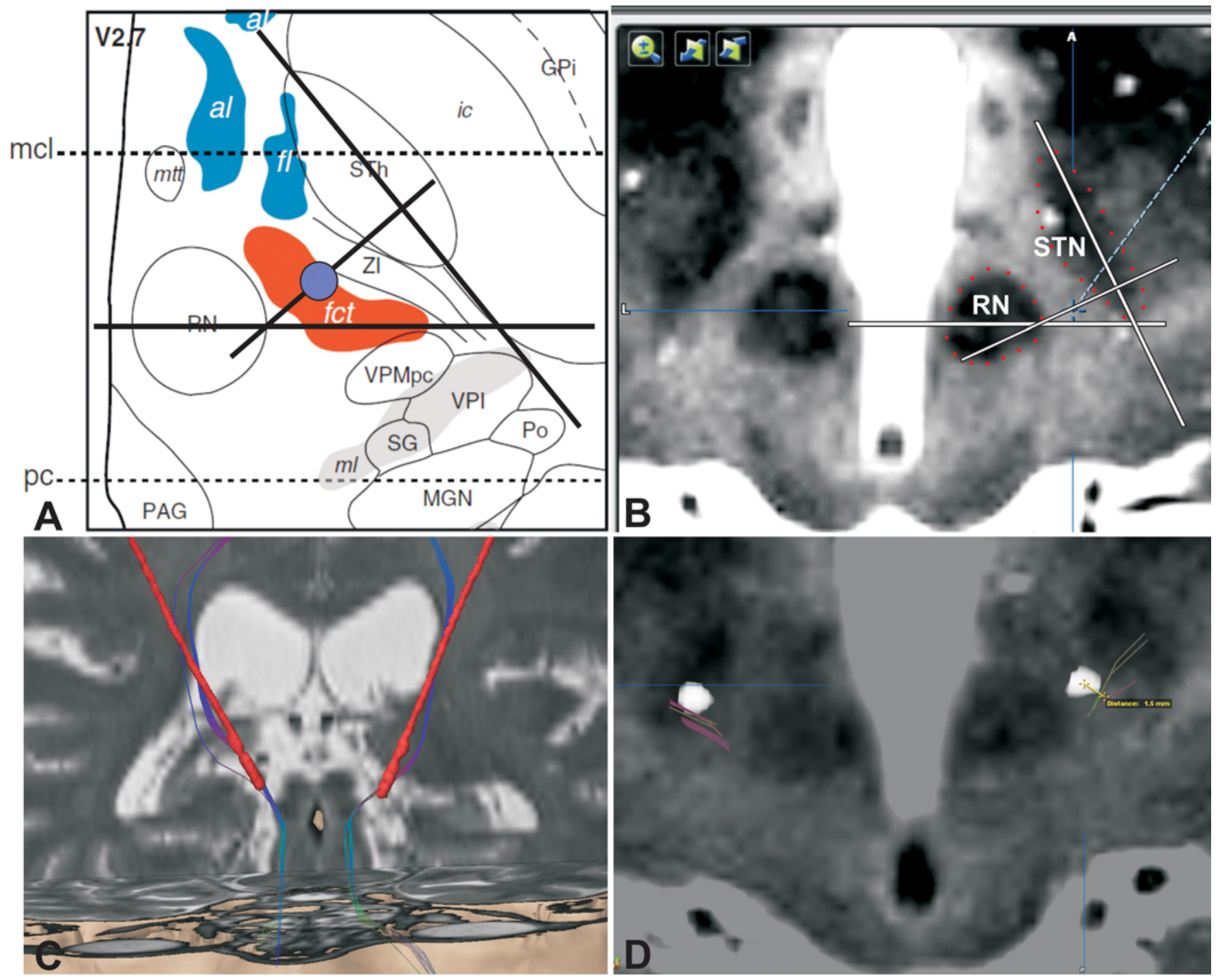

FIG. 1. Targeting strategy for the PSA and determination of the distance between the SC and the center of gravity of the DRTT. The theoretical target point at the center of the PSA is based on the stereotactic atlas of Morel (A). The applied targeting strategy based on 3-T T2-weighted MRI sequences leads to the planned target (B). The distance between the SC and the center of the tractography-based DRTT (1.5 mm in this case) was measured with iPlan 3.0 software $(\mathbf{C}$ and $\mathbf{D})$. al = ansa lenticularis; fct = cerebellothalmic fascicle; $\mathrm{fl}$ = fasciculus lenticularis; $\mathrm{GPi}=$ globus pallidus interna; ic = internal capsule; $\mathrm{mcl}=$ midcommissural line; $\mathrm{MGN}=$ medial geniculate nucleus; $\mathrm{ml}=$ medial lemniscus; $\mathrm{mtt}=$ mammillothalamic tract; $\mathrm{PAG}=$ periaqueductal gray; $\mathrm{pc}=$ posterior commissure; Po = posterior nucleus; $R N=$ red nucleus; $S G$ = suprageniculate nucleus; $S T h=$ subthalamic nucleus; VPI = ventral posterior inferior nucleus; VPMpc = ventral posterior medial nucleus, parvocellular division; ZI = zona incerta. Panel $\mathrm{A}$ modified from Gallay MN, Jeanmonod D, Liu J, Morel A: Human pallidothalamic and cerebellothalamic tracts: anatomical basis for functional stereotactic neurosurgery. Brain Struct Funct 212:443-463, 2008, with kind permission of the authors. Copyright the authors. CC BY-NC (https://creativecommons.org/licenses/by-nc/3.0/).

point (MCP) were determined as described previously by our group. ${ }^{28}$ Mean target coordinates, lead tip coordinates, and SC coordinates were projected onto axial and sagittal planes of the Morel stereotactic atlas..$^{13}$

On axial planes, the smallest distance from the center of the SC to the center of gravity of the DTI-based depicted DRTT was measured.

\section{Clinical Assessment}

Preoperative "medication-off" and postoperative "stim- ulation-on, medication-off" tremor severities were assessed "nonblinded" based on the Fahn-Tolosa-Marin Tremor Rating Scale (TRS) by specialized movement disorder neurologists at 6 and 12 months postoperatively. ${ }^{38}$ Two patients were lost to the 12-month follow-up as they were followed up in another clinic, but their 6-month follow-up data were available and used in our final analysis. The part A and part B subscores were determined separately for each hemibody. At each follow-up, side effects attributable to stimulation were recorded. We determined the difference 
TABLE 1. Clinical and demographic characteristics of the study cohort

\begin{tabular}{|c|c|c|c|c|c|c|c|c|c|c|c|c|c|c|}
\hline \multirow{3}{*}{$\begin{array}{c}\text { Patient } \\
\text { No. }\end{array}$} & \multirow{3}{*}{$\begin{array}{l}\text { Age } \\
(y r s)^{*}\end{array}$} & \multicolumn{6}{|c|}{ Stimulus Parameter } & \multicolumn{4}{|c|}{ \% Improvement } & \multicolumn{2}{|c|}{ SC Location } & \multirow[b]{3}{*}{ Side Effects } \\
\hline & & \multicolumn{2}{|c|}{ Amplitude (mA) } & \multicolumn{2}{|c|}{ Pulse Width ( $\mu \mathrm{sec})$} & \multicolumn{2}{|c|}{ Frequency $(\mathrm{Hz})$} & \multirow[b]{2}{*}{ TRS A } & \multirow[b]{2}{*}{ TRS B } & \multirow[b]{2}{*}{ TRS C } & \multirow[b]{2}{*}{ TRS Total } & \multirow[b]{2}{*}{$\mathrm{Lt}$} & \multirow[b]{2}{*}{ Rt } & \\
\hline & & $\mathrm{Lt}$ & Rt & $\mathrm{Lt}$ & $\mathrm{Rt}$ & $\mathrm{Lt}$ & Rt & & & & & & & \\
\hline 1 & 73 & 1.7 & 1.8 & 60 & 60 & 130 & 130 & 16.7 & 52 & 70.6 & 39.0 & $\mathrm{Zl}$ & $\mathrm{Zl} / \mathrm{fct}$ & None \\
\hline 2 & 53 & 1.6 & 2.1 & 60 & 60 & 130 & 130 & 66.7 & 18.2 & 50 & 36.3 & fct & fct & Paresthesia \\
\hline 3 & 80 & 2.3 & 1.9 & 60 & 60 & 130 & 130 & 32 & 63 & 62.5 & 52.6 & fct & $\mathrm{Zl} / \mathrm{fct}$ & Mild dysarthria \\
\hline 4 & 79 & 1.6 & 1.5 & 60 & 60 & 120 & 120 & 47.6 & 70.6 & 60 & 58.6 & fct & fct & None \\
\hline 5 & 69 & 1.6 & 1.5 & 60 & 60 & 130 & 130 & 75 & 70 & 84.6 & 75.6 & fct & fct & None \\
\hline 6 & 73 & 2.3 & 2.9 & 60 & 60 & 130 & 130 & 100 & 80 & 92.3 & 89.2 & $\mathrm{Zl}$ & $\mathrm{ZI}$ & None \\
\hline 7 & 71 & 1.5 & 4 & 60 & 60 & 119 & 119 & 75 & 16.7 & 58.3 & 50.0 & $\mathrm{ZI}$ & fct & Dysarthria \\
\hline 8 & 35 & 2.7 & 2 & 60 & 60 & 149 & 149 & 42.8 & 0 & 100 & 56.3 & $\mathrm{Zl}$ & $\mathrm{Zl}$ & Mild dysarthria \\
\hline 9 & 72 & 3.7 & 2.6 & 60 & 60 & 136 & 136 & 23.1 & 29.4 & 69.2 & 39.5 & $\mathrm{ZI}$ & $\mathrm{Zl}$ & None \\
\hline 10 & 53 & 2.6 & 2.3 & 60 & 60 & 185 & 185 & 50 & 54.5 & 90.9 & 61.7 & $\mathrm{Zl}$ & Vim & None \\
\hline 11 & 80 & 2 & 3.5 & 60 & 60 & 185 & 185 & 58.8 & 58.6 & 43.7 & 54.8 & fct & Vim & Mild dysarthria \\
\hline
\end{tabular}

${ }^{*}$ Age at surgery.

between each corresponding pre- and postoperative TRS subscore (part A, part B, part C, and total).

We determined the difference in the mean stereotactic SC position between the more effective SCs (defined as percentage improvement of part A and part B TRS subscores for the corresponding contralateral hemibody greater than $60 \%$ ) and less effective SCs (defined as percentage improvement of part A and part B TRS subscores for the corresponding contralateral hemibody less than 30\%).

\section{Outcome Measures and Statistical Analysis}

The primary outcome was the difference of means between preoperative "medication-off" and postoperative

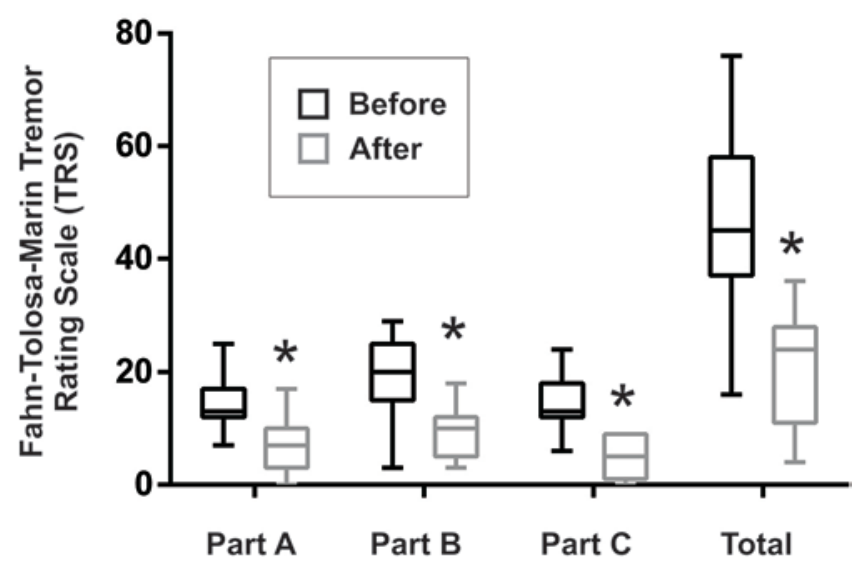

FIG. 2. Box and whiskers plot showing the pre- and postoperative total scores and subscores from the Fahn-Tolosa-Marin Tremor Rating Scale (TRS). Part A scores improved from $14.3 \pm 5.2$ to $7 \pm 4.7$, part B scores improved from $19 \pm 7.4$ to $9.2 \pm 4.6$, and part $C$ scores improved from $14.8 \pm 4.9$ to $4.8 \pm 3.5$ postoperatively. There was a statistically significant reduction of the total TRS and the 3 TRS subscores $(p<$ 0.001; 2-way repeated measures ANOVA). The boxes indicate IQRs, the horizontal lines within the boxes indicate the median values, and the whiskers indicate the minimum and maximum values. "medication-off, stimulation-on" TRS total scores. Secondary outcomes were the difference of means between preoperative "medication-off" and postoperative "medication-off, stimulation-on" TRS subscores; the mean target and SC position with reference to the MCP and DRTT; and the difference in the mean stereotactic SC position and distance to DRTT between the most and least effective SCs.

Data were analyzed with descriptive/parametric statistics using Prism software (GraphPad Prism 6). The Shapiro-Wilk normality test was used to test for normal distribution of data sets. Student's unpaired t-test was applied to compare the mean coordinates of independent groups. One-way ANOVA was applied to compare preand postoperative tremor subscores. Data are presented as mean \pm standard deviation or $95 \%$ confidence interval. Correlation analysis was tested by Spearman's R and linear regression analysis. A $p$ value $<0.05$ was considered statistically significant.

\section{Results \\ Clinical Outcome Assessment}

Table 1 summarizes the stimulation parameters as well as the percentage improvement in TRS subscores for each patient. The mean preoperative global TRS score was 47.2 \pm 15.7. The mean postoperative TRS score was $21.3 \pm$ 10.7, demonstrating a significant reduction. The mean percentage improvement was $55.1 \%$ (95\% CI 30.9\%-59.3\%). Figure 2 shows the mean pre- and postoperative TRS subscores for the study cohort. The most common stimulation-induced side effects were dysarthria (36\%) and paresthesia (9\%). No postoperative hemorrhage, infection, granuloma, or DBS device malfunction occurred within the 12-month follow-up time in our series of patients.

\section{Target, Lead Tip, and SC Position}

The mean preoperative target coordinates were LAT $10.57 \pm 0.81$, AP $-5.20 \pm 0.71$, and VERT $-3.17 \pm 0.53$ 


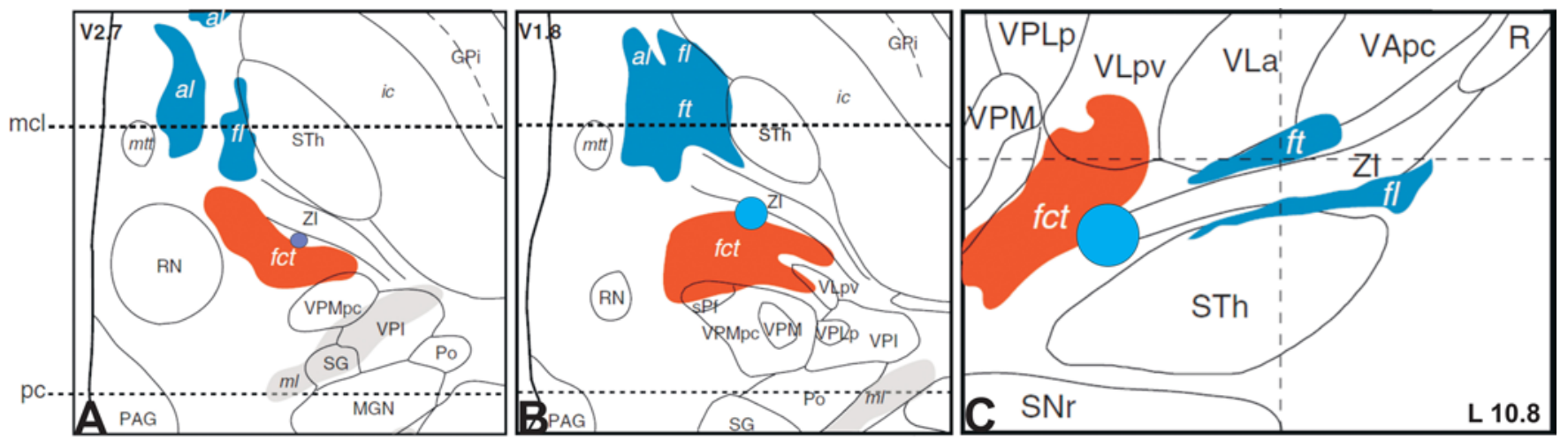

FIG. 3. Mean target (A) and SC (B and C) position projected onto the stereotactic atlas of Morel. The mean target point (A) was slightly posterior to the theoretically expected target point according to MRI-based anatomical landmarks and projected onto the center of the PSA at the margin of the cerebellothalamic fascicle (fct) and zona incerta (ZI). The mean SC position was superior with respect to the planned target and projected onto the PSA at the margin of the fct and ZI on both axial (B) and sagittal (C) images. $\mathrm{ft}=$ fasciculus thalamicus; $\mathrm{R}=$ reticular thalamic nucleus; $\mathrm{sPf}=$ supraparafascicular nucleus; $\mathrm{VApc}=$ ventral anterior nucleus, parvocellular division; VLpv = ventral lateral posterior nucleus, ventral division; VPLp = ventral posterior lateral nucleus, posterior division; VPM = ventral posterior medial nucleus. Adapted from Gallay MN, Jeanmonod D, Liu J, Morel A: Human pallidothalamic and cerebellothalamic tracts: anatomical basis for functional stereotactic neurosurgery. Brain Struct Funct 212:443-463, 2008, with kind permission of the authors. Copyright the authors. CC BY-NC (https://creativecommons.org/licenses/by-nc/3.0/).

$\mathrm{mm}$. Projected onto the stereotactic atlas of Morel, the mean target position showed a good correspondence with the intended target based on MRI anatomical landmarks (Fig. 3A).

The mean vector of error between the intended target and actual lead tip position was $1.01 \pm 1.10 \mathrm{~mm}$. The mean SC coordinates were LAT $10.92 \pm 1.50, \mathrm{AP}-3.92 \pm 0.97$, and VERT $-1.85 \pm 1.37 \mathrm{~mm}$. The mean SC position projected onto the anterior aspect of the fct at the margin of the ZI (Fig. 3B and C).

\section{More and Less Effective SC Positions}

The mean stereotactic coordinates for the more effective SC (leading to more than $60 \%$ TRS part A and B improvement on the contralateral side) were LAT 10.54 \pm 0.71 , AP $-4.32 \pm 0.64$, and VERT $-2.14 \pm 1.50 \mathrm{~mm}$. The mean stereotactic coordinates for the less effective SC (leading to less than 30\% TRS part A and B improvement on the contralateral side) were LAT $10.08 \pm 1.70$, AP $-4.46 \pm 0.72$, and VERT $-2.32 \pm 1.92 \mathrm{~mm}$. There was no statistically significant difference between the most and least efficient SC LAT, AP, and VERT coordinates, and both projected onto the atlas-based border of the fct and ZI (data not shown).

\section{DRTT Tractography Results}

The DRTT could not be tracked in an anatomically plausible way in 4 patients (4 hemispheres, 36\%). The mean distance of the SC to the center of the DRTT tractography model was $1.29 \pm 1.22 \mathrm{~mm}$. The most and least efficient SC showed no difference between their distance to the tracked DRTT $(1.84 \pm 1.24$ vs $0.92 \pm 1.21 \mathrm{~mm}, \mathrm{p}=$ $0.22)$.

\section{Discussion}

Our targeting approach to the PSA is based on anatom- ical landmarks in combination with MER showing a typical pattern of missing spiking activity to confirm targeting outside the STN and red nucleus. The results show that application of this technique leads to precise and accurate positioning of the DBS lead within the PSA with a small mean targeting error of $1.01 \mathrm{~mm}$. Projected onto the stereotactic atlas of Morel, the mean SC position lies within the PSA at the margin of the fct and ZI. Furthermore, we can show that bilateral DBS of the PSA is clinically effective, leading to a mean tremor reduction of $55.1 \%$ based on the TRS. Despite the well-known technical limitations of DTI-based tractography, our tractography results show that the SC position is in close spatial proximity to the tracked DRTT. We could not demonstrate a difference in position between the SCs that was clinically more or less effective based on stereotactic and atlas data or tractography results.

\section{Clinical Outcome}

Our study was motivated by previous work of different groups presenting good outcomes of PSA DBS for tremor. ${ }^{5,7,17,26,31-33}$ Until recently, only 5 studies included ET patients exclusively. Murata et al. presented a series of 8 ET patients who underwent unilateral DBS of the PSA with a mean reduction of $81 \%$ on a modified tremor scale. ${ }^{26}$ Plaha et al. reported on 4 ET patients with bilateral PSA DBS who had a mean reduction of $80.1 \%$ based on the TRS. ${ }^{33}$ Later, the same group presented results for ET patients in whom DBS was targeted more specifically in the caudal ZI. The authors reported a mean reduction of $75.9 \%$ in 6 patients and $73.8 \%$ in 15 patients after a mean followup of 32 months. ${ }^{31,32}$ Blomstedt and coworkers presented the largest series of patients undergoing unilateral DBS of the PSA for treatment of ET (21 patients). According to their results, a tremor reduction of $60 \%$ on the TRS after 1 year of clinical follow-up was achieved. ${ }^{5}$ The mean tremor reduction of 55\% in our cohort is in line with previous 


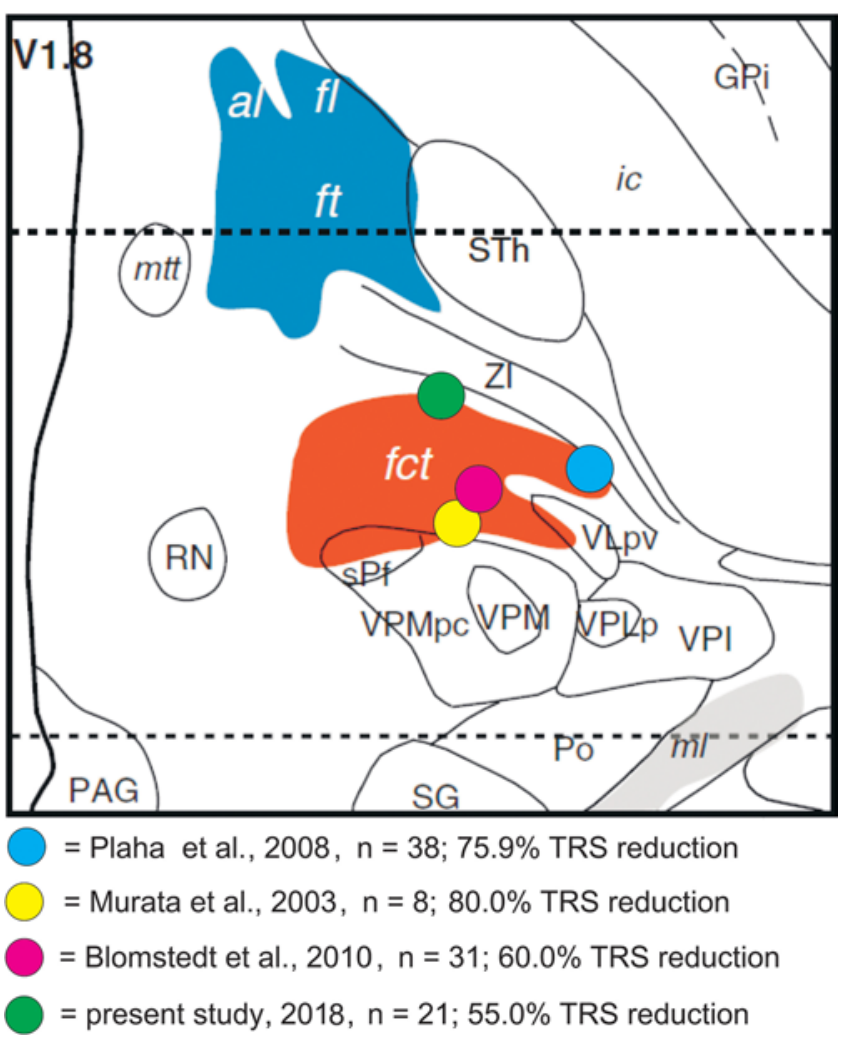

FIG. 4. Comparison of the mean SC position. The stereotactic coordinates of the mean SC position of our results and those of other groups are projected onto the stereotactic atlas of Morel. Modified from Gallay MN, Jeanmonod D, Liu J, Morel A: Human pallidothalamic and cerebellothalamic tracts: anatomical basis for functional stereotactic neurosurgery. Brain Struct Funct 212:443-463, 2008, with kind permission of the authors. Copyright the authors. CC BY-NC (https://creativecommons. org/licenses/by-nc/3.0/).

studies. No relevant complication occurred in our series of patients. However, $36 \%$ of the patients showed stimulation-induced mild dysarthria, which prevented application of higher stimulation amplitudes and thus limited further tremor suppression.

\section{Optimal Stimulation Site}

The PSA contains the zona incerta (ZI) as well as white matter tracts considered the prelemniscal radiation (RaprL) and fields of Forel (fields $\mathrm{H} 1$ and $\mathrm{H} 2$ ). Whereas the RaprL contains fct, the fields of Forel consist of the ansa lenticularis and fasciculus lenticularis, which form pallidothalamic fibers. ${ }^{12}$ Thus, the PSA contains different fiber pathways that convey information within cortico-cerebello-thalamic and cortico-striato-pallido-thalamic loops. The anatomical complexity of the PSA will have implications on the neuromodulatory effects of DBS in this area and might determine the relationship between the exact lead location and the therapeutic window in each patient. In previous studies the exact SC position within the PSA differed significantly between groups. ${ }^{5,26,30}$ Despite the fact that different subareas of the PSA seemed to be stimulated, our group has previously suggested that the DRTT might be the common stimulated anatomical structure. ${ }^{12}$
Figure 4 reviews the mean SC positions of the above-cited studies (if applicable) and our results projected onto the stereotactic atlas of Morel. The mean SC position of each of the cited studies covers the fct, but at the margin of the ZI in the case of findings by Plaha et al. ${ }^{32}$ and our group. The hypothesis that the DRTT might mediate the stimulation-induced tremor-suppressive effect is also postulated by recent work based on DTI tractography. ${ }^{9,11}$ On the other hand, Plaha et al. suggested that stimulation of the ZI is effective to suppress tremor in ET patients. The ZI is a heterogeneous reticular nucleus of primarily GABAergic neurons with reciprocal connections to the basal ganglia, cerebellum, thalamus, and brainstem nuclei..$^{18,23,34,35} \mathrm{ZI}$ neurons probably play a role in mediating neuronal synchronization in the subcortical motor network, resulting in tremor oscillations. ${ }^{16,32}$

To confine the optimal stimulation site within the PSA subregions in our series of patients, we analyzed the anatomical position of the clinically most effective SCs and compared these positions to the position of the least effective SCs. No significant differences were found between the most and least effective SC positions and their distance to the tractography-based DRTT. Thus, the question of an optimal stimulation site within the PSA remains unanswered at this point and needs to be addressed in future studies with a bigger sample size and stimulation field models.

\section{Limitations}

Our study has some limitations. First, the sample size is too small to allow generalization of our results to a broader population. Second, the postoperative clinical testing was not performed in a blinded manner. Third, we have not included the analysis of stimulation volumes based on stimulation field models such as proposed by McIntyre and coworkers or Butson and coworkers to further define the volume of tissue activated., 6,21,22 Furthermore, we implanted 2 different types of DBS leads in our study population. As there is no study directly comparing constant voltage source-driven $(\mathrm{CV})$ omnidirectional DBS leads with constant current source-driven (CC) directional DBS leads, we cannot exclude the introduction of a potential bias. Our targeting approach was based on anatomical landmarks visible on T2-weighted MRI rather than tractography of the DRTT. Despite the application of tractography-guided targeting of the DRTT by several groups, its accuracy for displaying the anatomical DRTT has yet to be validated. Only probabilistic DTI-based tractography of the DRTT has so far been validated in a postmortem anatomical study, with 7-T MRI revealing moderate concordance of the tracked and anatomical DRTT at the level of the midbrain. ${ }^{24}$ Moreover, application of deterministic DTI-based tractography to display the DRTT has some limitations that shall be mentioned only cursorily. The relatively low resolution of our DTI sequences and the small number of gradients that we used (only 12) in order to achieve compatibility with the Brainlab software algorithm has implications for the reliability of the DRTT tractography results. The tensor calculation model fails in cases of voxels containing 2 or more fiber tracts with different orientations. These theoretical limitations are practically relevant and are reflected by the 
high percentage (36\%) of anatomically implausible tracking results. This reflects the main reason why we prefer to rely on anatomical landmarks rather than tractography as a targeting strategy for the PSA. The question of whether application of higher angular resolution published by other groups is more accurate remains to be determined. . $^{8,36}$

Due to these limitations, our results do not allow reliable conclusions about an optimal stimulation target within the PSA and whether this is confined to one of its substructures. Furthermore, due to the study design our results do not contribute to disentangling one of the most relevant questions-whether there are differences between Vim and PSA targeting for ET in terms of clinical outcome and side effects.

\section{Conclusions}

Targeting of the PSA based on anatomical landmarks on MRI is accurate and leads to good long-term clinical results. Although our results support the concept of the DRTT to mediate the tremor-suppressive effect of DBS, the optimal stimulation site within the PSA still has to be further defined in future studies of directional leads and reliable electric field models.

\section{References}

1. Bastin ME, Armitage PA, Marshall I: A theoretical study of the effect of experimental noise on the measurement of anisotropy in diffusion imaging. Magn Reson Imaging 16:773-785, 1998

2. Bejjani BP, Dormont D, Pidoux B, Yelnik J, Damier P, Arnulf I, et al: Bilateral subthalamic stimulation for Parkinson's disease by using three-dimensional stereotactic magnetic resonance imaging and electrophysiological guidance. J Neurosurg 92:615-625, 2000

3. Benabid AL, Benazzouz A, Hoffmann D, Limousin P, Krack P, Pollak P: Long-term electrical inhibition of deep brain targets in movement disorders. Mov Disord 13 (Suppl 3):119-125, 1998

4. Benabid AL, Pollak P, Gao D, Hoffmann D, Limousin P, Gay E, et al: Chronic electrical stimulation of the ventralis intermedius nucleus of the thalamus as a treatment of movement disorders. J Neurosurg 84:203-214, 1996

5. Blomstedt P, Sandvik U, Tisch S: Deep brain stimulation in the posterior subthalamic area in the treatment of essential tremor. Mov Disord 25:1350-1356, 2010

6. Butson CR, Maks CB, McIntyre CC: Sources and effects of electrode impedance during deep brain stimulation. Clin Neurophysiol 117:447-454, 2006

7. Carrillo-Ruiz JD, Velasco F, Jimènez F, Castro G, Velasco AL, Hernández JA, et al: Bilateral electrical stimulation of prelemniscal radiations in the treatment of advanced Parkinson's disease. Neurosurgery 62:347-359, 2008

8. Coenen VA, Allert N, Mädler B: A role of diffusion tensor imaging fiber tracking in deep brain stimulation surgery: DBS of the dentato-rubro-thalamic tract (drt) for the treatment of therapy-refractory tremor. Acta Neurochir (Wien) 153:1579-1585, 2011

9. Coenen VA, Allert N, Paus S, Kronenbürger M, Urbach H, Mädler B: Modulation of the cerebello-thalamo-cortical network in thalamic deep brain stimulation for tremor: a diffusion tensor imaging study. Neurosurgery 75:657-670, 2014

10. Deuschl G, Bain P, Brin M: Consensus statement of the Movement Disorder Society on tremor. Mov Disord 13 (Suppl 3):2-23, 1998
11. Fenoy AJ, Schiess MC: Deep brain stimulation of the dentato-rubro-thalamic tract: outcomes of direct targeting for tremor. Neuromodulation 20:429-436, 2017

12. Fiechter M, Nowacki A, Oertel MF, Fichtner J, Debove I, Lachenmayer ML, et al: Deep brain stimulation for tremor: is there a common structure? Stereotact Funct Neurosurg 95:243-250, 2017

13. Gallay MN, Jeanmonod D, Liu J, Morel A: Human pallidothalamic and cerebellothalamic tracts: anatomical basis for functional stereotactic neurosurgery. Brain Struct Funct 212:443-463, 2008

14. Hariz MI, Shamsgovara P, Johansson F, Hariz G, Fodstad H: Tolerance and tremor rebound following long-term chronic thalamic stimulation for Parkinsonian and essential tremor. Stereotact Funct Neurosurg 72:208-218, 1999

15. Hassler R: Anatomy of the thalamus, in Schaltenbrand G, Bailey P (eds): Introduction to Stereotaxic Operations With an Atlas of the Human Brain. Stuttgart: Thieme, 1959, pp 230-290

16. Kim U, Sanchez-Vives MV, McCormick DA: Functional dynamics of GABAergic inhibition in the thalamus. Science 278:130-134, 1997

17. Kitagawa M, Murata J, Kikuchi S, Sawamura Y, Saito H, Sasaki H, et al: Deep brain stimulation of subthalamic area for severe proximal tremor. Neurology 55:114-116, 2000

18. Kolmac CI, Power BD, Mitrofanis J: Patterns of connections between zona incerta and brainstem in rats. J Comp Neurol 396:544-555, 1998

19. Limousin P, Speelman JD, Gielen F, Janssens M: Multicentre European study of thalamic stimulation in parkinsonian and essential tremor. J Neurol Neurosurg Psychiatry 66:289296, 1999

20. Lozano AM: Vim thalamic stimulation for tremor. Arch Med Res 31:266-269, 2000

21. McIntyre CC, Grill WM: Excitation of central nervous system neurons by nonuniform electric fields. Biophys J 76:878-888, 1999

22. McIntyre CC, Mori S, Sherman DL, Thakor NV, Vitek JL: Electric field and stimulating influence generated by deep brain stimulation of the subthalamic nucleus. Clin Neurophysiol 115:589-595, 2004

23. Mitrofanis J: Some certainty for the "zone of uncertainty"? Exploring the function of the zona incerta. Neuroscience 130:1-15, 2005

24. Mollink J, van Baarsen KM, Dederen PJ, Foxley S, Miller KL, Jbabdi S, et al: Dentatorubrothalamic tract localization with postmortem MR diffusion tractography compared to histological 3D reconstruction. Brain Struct Funct 221:3487-3501, 2016

25. Mori S, Zhang J: Principles of diffusion tensor imaging and its applications to basic neuroscience research. Neuron 51:527-539, 2006

26. Murata J, Kitagawa M, Uesugi H, Saito H, Iwasaki Y, Kikuchi S, et al: Electrical stimulation of the posterior subthalamic area for the treatment of intractable proximal tremor. J Neurosurg 99:708-715, 2003

27. Nowacki A, Debove I, Fiechter M, Rossi F, Oertel MF, Wiest R, et al: Targeting accuracy of the subthalamic nucleus in deep brain stimulation surgery: comparison between $3 \mathrm{~T}$ T2weighted magnetic resonance imaging and microelectrode recording results. Oper Neurosurg (Hagerstown) 15:66-71, 2018

28. Nowacki A, Fiechter M, Fichtner J, Debove I, Lachenmayer L, Schüpbach M, et al: Using MDEFT MRI sequences to target the GPi in DBS surgery. PLoS One 10:e0137868, 2015

29. Pahwa R, Lyons KL, Wilkinson SB, Carpenter MA, Tröster AI, Searl JP, et al: Bilateral thalamic stimulation for the treatment of essential tremor. Neurology 53:1447-1450, 1999

30. Pinsker MO, Volkmann J, Falk D, Herzog J, Alfke K, 
Steigerwald F, et al: Electrode implantation for deep brain stimulation in dystonia: a fast spin-echo inversion-recovery sequence technique for direct stereotactic targeting of the GPI. Zentralbl Neurochir 69:71-75, 2008

31. Plaha P, Javed S, Agombar D, O' Farrell G, Khan S, Whone A, et al: Bilateral caudal zona incerta nucleus stimulation for essential tremor: outcome and quality of life. J Neurol Neurosurg Psychiatry 82:899-904, 2011

32. Plaha P, Khan S, Gill SS: Bilateral stimulation of the caudal zona incerta nucleus for tremor control. J Neurol Neurosurg Psychiatry 79:504-513, 2008

33. Plaha P, Patel NK, Gill SS: Stimulation of the subthalamic region for essential tremor. J Neurosurg 101:48-54, 2004

34. Power BD, Mitrofanis J: Ultrastructure of afferents from the zona incerta to the posterior and parafascicular thalamic nuclei of rats. J Comp Neurol 451:33-44, 2002

35. Roger M, Cadusseau J: Afferents to the zona incerta in the rat: a combined retrograde and anterograde study. J Comp Neurol 241:480-492, 1985

36. Sammartino F, Krishna V, King NK, Lozano AM, Schwartz ML, Huang Y, et al: Tractography-based ventral intermediate nucleus targeting: novel methodology and intraoperative validation. Mov Disord 31:1217-1225, 2016

37. Schlaier J, Anthofer J, Steib K, Fellner C, Rothenfusser E, Brawanski A, et al: Deep brain stimulation for essential tremor: targeting the dentato-rubro-thalamic tract? Neuromodulation 18:105-112, 2015

38. Stacy MA, Elble RJ, Ondo WG, Wu SC, Hulihan J: Assessment of interrater and intrarater reliability of the FahnTolosa-Marin Tremor Rating Scale in essential tremor. Mov Disord 22:833-838, 2007

39. Wertheimer P, Lapras C, Levy A: [Trials in thalamic surgery.] Neurochirurgie 6:105-112, 1960 (Fr)
40. Yousry TA, Schmid UD, Alkadhi H, Schmidt D, Peraud A, Buettner A, et al: Localization of the motor hand area to a knob on the precentral gyrus. A new landmark. Brain 120:141-157, 1997

\section{Disclosures}

Andreas Nowacki reports being invited to research meetings sponsored by Boston Scientific not related to the current work. Ines Debove reports receiving financial support from Boston Scientific not related to the current work. Frédéric Rossi reports receiving financial support from Medtronic not related to the current work. Michael Schüpbach reports consultant relationships with Medtronic, Boston Scientific, and Aleva. Claudio Pollo reports a consultant relationship with Boston Scientific.

\section{Author Contributions}

Conception and design: Pollo. Acquisition of data: Nowacki, Debove, Rossi, Schlaeppi, Petermann, Wiest, Schüpbach. Analysis and interpretation of data: Nowacki, Pollo. Drafting the article: Nowacki, Pollo. Critically revising the article: Debove, Rossi, Wiest, Schüpbach. Approved the final version of the manuscript on behalf of all authors: Nowacki. Statistical analysis: Nowacki. Administrative/technical/material support: Petermann, Wiest. Study supervision: Pollo.

\section{Correspondence}

Andreas Nowacki: Inselspital, University Hospital Bern, University of Bern, Switzerland. neuro.nowacki@gmail.com. 